\title{
EFEITO DA APLICAÇÃO DA URÉIA EM DIFERENTES DOSES E ÉPOCAS SOBRE UM HÍBRIDO DE MILHO PARA SILAGEM
}

ATHAYDE, Antônio Augusto Rocha ${ }^{1}$

\begin{abstract}
RESUMO: A cultura do milho apresenta elevado potencial na produção de grãos, entretanto no Brasil alguns fatores restrigem a expressão da capacidade máxima de produtividade da cultura. A obtenção de produtividades economicamente viáveis, demandam uma nutrição mineral adequada e o nitrogênio é o nutriente que, modula, com maior efeito esta produção. O objetivo do trabalho foi comparar os efeitos de diferentes doses e época de aplicação de uréia em cobertura, no município de Bambuí-MG. O trabalho foi desenvolvido no setor de produção do Instituto Federal de Minas Gerais - Campus Bambuí, em Latossolo Vermelho Distrófico, no ano agrícola 2010/11. O Delineamento experimental utilizado foi de blocos casualizados em esquema fatorial de parcelas subdivididas, sendo 4 doses de $\mathrm{N}\left(100,150,200\right.$ e $\left.250 \mathrm{Kg}_{\text {de }} \mathrm{N} \mathrm{ha}^{-1}\right)$ na parcela e 3 épocas diferentes de cobertura (21, 28 e 35 dias após o plantio) na sub parcela, com quatro repetições. Todas as aplicações foram realizadas manualmente e a fonte de $\mathrm{N}$ utilizado foi a Uréia $(45 \%$ N). Foram verificados que para o híbrido AG 1051, cultivado na região de Bambuí destinado a produção de silagem a dose recomendada pela CFSEMG (1999) foi suficiente para suprir as demandas nutricionais do híbrido e obter a produtividade estimada, uma vez que não houve respostas do híbrido em função das doses aplicadas. Nos parâmetros avaliados, as épocas de aplicação também não influenciaram de forma significativa, os parâmetros avaliados, com exceção na altura quando foi aplicada a dose de $200 \mathrm{~kg} \mathrm{ha}^{-1}$ onde observou-se um maior incremento na aplicação realizada aos 28 dias.
\end{abstract}

Palavras-chave: Adubação. Cobertura. Nitrogênio. Zea mays

\section{EFFECT OF UREA AT DIFFERENT DOSES AND TIME ABOUT THE HYBRID OF CORN FOR SILAGE}

\begin{abstract}
SUMMARY : Corn has a high potential in grain production in Brazil but some factors restrict the expression of maximum yield. The achievement of economically viable products, require an adequate mineral nutrition and nitrogen is the nutrient that modulates, with greater effect this production. The objective of this study was to compare the effects of different doses and timing of urea application in coverage in the town of Bambuí-MG. The work was developed in the production sector of the Federal Institute of Minas Gerais - Campus Bambuí, in a Typic Dystrophic in crop year 2010/11. The experimental design was randomized blocks in split plots, with four $\mathrm{N}$ rates $(100,150,200$ and $250 \mathrm{~kg} \mathrm{~N}$ ha-1) in the plot and cover three different times (21, 28 and 35 days after planting) in the sub plot with four replications. All applications were done manually and the $\mathrm{N}$ source used was urea $(45 \% \mathrm{~N})$. We checked that for the hybrid AG 1051, grown in the region of Bambuí for the production of silage by CFSEMG the recommended dose (1999) was sufficient to meet the nutritional demands of the hybrid and the estimated productivity gain, since there were no responses of the hybrid depending on the doses applied. In the parameters evaluated, the application time did not influence significantly the parameters evaluated, except at the time when it was applied a dose of $200 \mathrm{~kg}$ ha- 1 where there was a greater increase in application performed at 28 days.
\end{abstract}

Keywords: Fertilization. Cover. Nitrogen. Zea mays

\section{INTRODUÇÃO}

O milho Zea mays L. é um cereal importante sob os aspectos econômicos e sociais e uma das culturas mais estudadas pelo alto valor nutricional dos grãos, e sua importância na alimentação humana,

\footnotetext{
${ }^{1}$ Professor MEC/ INSTITUTO FEDERAL DE EDUCAÇÃO CIÊNCIA E TECNOLOGIA DE MINAS GERAIS CAMPUS BAMBUÍ. Zootecnista (UFLA), 1990, Mestre em Zootecnia (UFLA) 1996, Doutor em Zootecnia Forragicultura e Pastagens (UFLA) 2010. Experiência didática nas áreas; Bovinocultura (Leite e corte), Nutrição animal, Conservação de Plantas Forrageiras, Extensão Rural, Inovação e Propriedade Intelectual. Atuação docente nas áreas; Bovinocultura, Piscicultura, Pesquisa Tecnológica, Qualidade de Água e Meio Ambiente. Doutor em Zootecnia: Forragicultura e Pastagens/UFLA/2010.
} 
animal e produção de matérias-primas à indústria (FANCELLI ; LIMA, 1989).

O Brasil ocupa a terceira posição entre os maiores produtores do mundo. Segundo CONAB (2010), a área cultivada no Brasil na safra 2009/2010 foi de 12.932,4 mil hectares, sendo o Mato Grosso o estado maior produtor de milho do país. Mesmo considerado um dos maiores produtores mundiais, a produtividade média do país em torno de $4.412 \mathrm{~kg} / \mathrm{ha}$ de milho safra, é considerada baixa.

$\mathrm{O}$ nitrogênio $(\mathrm{N})$ é o nutriente mineral exigido em maior quantidade pelo milho, sendo ainda o maior limitador da produtividade de grãos, e em geral, é necessário, a utilização de uma adubação nitrogenada complementar aos teores fornecidos pelo solo, com vistas a obter produtividades elevadas. No Brasil, aproximados $47 \%$ do $\mathrm{N}$ consumido é na forma de uréia, $25 \%$ como sulfato de amônio e $22 \%$ como nitrato de amônio (POTAFOS, 2010).

Neste sentido, a uréia e o sulfato de amônio são as fontes de $\mathrm{N}$ mais utilizadas na atualidade. As exigências nutricionais são menores nos estádios iniciais de crescimento, entretanto, há indícios de que altas concentrações de $\mathrm{N}$ na zona radicular beneficiariam o rápido crescimento da planta nessa fase bem como promoveria aumentos na produtividade de grãos (YAMADA, 1996).

O estágio vegetativo V4 a V6, onde podem ser observadas de 4 a 6 folhas completamente formadas, é considerado o ponto definidor da produção da planta. Pela importância do suprimento do N no solo, a ocorrência de deficiência desse nutriente nessas fases reduz o número de óvulos nos primórdios da espiga (FANCELLI, 2000), reduzem a síntese de clorofila, com redução da taxa fotossintética, resultando em menor desenvolvimento e produtividade da planta.

O nitrogênio $(\mathrm{N})$ é um nutriente que apresenta problemas de manipulação, que podem concorrer para aumento de perdas causadas na aplicação, tais como a volatilização e a lixiviação. Em função dessas perdas na utilização e que o milho é uma das culturas mais responsivas em incrementos na produtividade em função da adubação nitrogenada, torna-se importantes ampliar os estudos em relação às épocas de aplicação e a dose do nutriente que resulta em maior produtividade. Os objetivos deste trabalho foram comparar o efeito da adubação com uréia em parâmetros produtivos do milho utilizando diferentes épocas de cobertura e diferentes doses de $\mathrm{N}$ aplicado.

\section{MATERIAL E MÉTODOS}

O experimento foi implantado em Dezembro de 2010, em campo experimental no Instituto Federal Minas Gerais (IFMG) - Campus Bambuí, coordenadas geográficas de -20 00' 23', de latitude e $45^{\circ} 58^{\prime} 37^{\prime}$ ' de longitude e $706 \mathrm{~m}$ de altitude, em área de Latossolo Vermelho Distroferrico textura argilosa.

O preparo do solo foi realizado pelo Sistema de Plantio Direto (SPD), com uma dessecação realizada em Novembro de 2010, sendo aplicados 4 litros de Glifosato ha ${ }^{-1}$ e 1,5 litros de 2,4D ha ${ }^{-1}$. A semente de milho utilizada foi o híbrido AG 1051. Este híbrido foi utilizado pela adaptação as condições regionais, sendo indicado para produção de silagem e milho verde com elevadas produtividades. O híbrido AG 1051 apresenta porte alto, ciclo semi-precoce, grão dentado amarelo, inserção de espiga alta, boa qualidade de colmo. O espaçamento utilizado no plantio foi $0,85 \mathrm{~m}$ entre linhas, com uso de 6 sementes metro linear ${ }^{-1}$, totalizando um stand de 70.588,24 plantas ha $^{-1}$. A adubação de plantio foi a mesma em todos os tratamentos, sendo aplicados $15 \mathrm{~kg}$ de $\mathrm{N}, 50 \mathrm{~kg}$ de $\mathrm{P}_{2} \mathrm{O}_{5}$ e $80 \mathrm{~kg}$ de $\mathrm{K}_{2} \mathrm{O}$ ha ${ }^{-1}$, em conformidade com a análise de solo (Tabela 1), realizada na área e as recomendações da CFSEMG (1999). 
Tabela 1- Características Químicas do solo avaliadas na camada de $0-20 \mathrm{~cm}$

\begin{tabular}{cccccccc}
\hline Área & $\mathrm{pH}$ & $\mathrm{P}$ & $\mathrm{K}$ & $\mathrm{Ca}$ & $\mathrm{Mg}$ & $\mathrm{Al}$ & $\mathrm{H}+\mathrm{Al}$ \\
\hline Milho antigo & & & & & & & \\
Café 0-20 & $\mathrm{H} 2 \mathrm{O}$ & $\mathrm{mg} / \mathrm{dm}^{3}$ & $\mathrm{mg} / \mathrm{dm}^{3}$ & $\mathrm{cmolc}^{2} \mathrm{dm}^{3}$ & $\mathrm{cmolc}^{2} \mathrm{dm}^{3}$ & $\mathrm{cmolc} / \mathrm{dm}^{3}$ & $\mathrm{cmolc} / \mathrm{dm}^{3}$ \\
& 5,6 & 9,5 & 120 & 2,86 & 0,78 & 0 & 3,91 \\
Milho antigo & & & & & & & \\
Café 0-20 & $\mathrm{SB}$ & $\mathrm{t}$ & $\mathrm{T}$ & $\mathrm{V}$ & $\mathrm{m}$ & $\mathrm{MO}$ & $\mathrm{P}-\mathrm{rem}$ \\
& $\mathrm{cmolc} / \mathrm{dm}^{3}$ & $\mathrm{cmolc} / \mathrm{dm}^{3}$ & $\mathrm{cmolc} / \mathrm{dm}^{3}$ & $\%$ & $\%$ & $\mathrm{dag} / \mathrm{kg}$ & $\mathrm{mg} / \mathrm{L}$ \\
& 3,95 & 3,95 & 7,86 & 50,25 & 0 & 2,4 & 8,2 \\
\hline
\end{tabular}

Adotou-se o delineamento experimental em blocos ao acaso em esquema fatorial de parcelas subdivididas, sendo 4 doses de $\mathrm{N}\left(100,150,200\right.$ e $\left.250 \mathrm{Kg}_{\text {de }} \mathrm{N} \mathrm{ha}^{-1}\right)$ na parcela e 3 épocas diferentes de cobertura $(21,28$ e 35 dias após o plantio) na sub parcela, em quatro repetições. As aplicações de adubação foram todas realizadas manualmente e a fonte de $\mathrm{N}$ a uréia $(45 \% \mathrm{~N})$. Cada parcela experimental foi composta por 5 metros de comprimento e 4 fileiras de milho, com uma área de $17 \mathrm{~m}^{2}$ por parcela. Cada bloco foi composto por 12 tratamentos e área total de $204 \mathrm{~m}^{2}$. Foi considerado como parcela útil as duas linhas centrais de cada parcela, considerando-se um metro para cada uma, sendo avaliadas 9 plantas por tratamento.

Os parâmetros avaliados foram; a altura da inserção da espiga, a altura da planta e a produtividade de massa para silagem em toneladas por hectare. A produtividade foi calculada pela projeção em área total, com a pesagem das plantas colhidas por tratamento e a extrapolação para um hectare. A altura total da planta e a inserção de espiga foram obtidos pela mensuração utilizando-se uma fita métrica antes da realização do corte das plantas. Os dados foram submetidos à análise de variância e as médias foram comparadas pelo teste de Tukey a 5\%, com a utilização do programa estatístico Sisvar 5.0 (FERREIRA, 2007).

\section{RESULTADOS E DISCUSSÃO}

Na Tabela 2 são apresentados os resultados da altura média da inserção das espigas em função dos diferentes tratamentos testados. Não foram verificados efeitos significativos em relação a este parâmetro. Neste sentido, as doses e as épocas de aplicação testadas foram estatisticamente semelhantes para esta variável.

Tabela 2 - Altura média da inserção da Espiga em função das diferentes doses de $\mathrm{N}$ e diferentes épocas de aplicação pós-plantio.

Dose de N Inserção da Espiga (m)

1 DIAS APÓS O PLANTIO ${ }^{1}$

\begin{tabular}{lccc} 
& 21 & 28 & 35 \\
\cline { 2 - 4 } $100 \mathrm{~kg} \mathrm{ha}^{-1}$ & $1,55 \mathrm{Aa}$ & $1,60 \mathrm{Aa}$ & $1,45 \mathrm{Aa}$ \\
$150 \mathrm{~kg} \mathrm{ha}^{-1}$ & $1,57 \mathrm{Aa}$ & $1,51 \mathrm{Aa}$ & $1,53 \mathrm{Aa}$ \\
$200 \mathrm{~kg} \mathrm{ha}^{-1}$ & $1,51 \mathrm{Aa}$ & $1,60 \mathrm{Aa}$ & $1,48 \mathrm{Aa}$ \\
$250 \mathrm{~kg} \mathrm{ha}^{-1}$ & $1,54 \mathrm{Aa}$ & $1,49 \mathrm{Aa}$ & $1,49 \mathrm{Aa}$ \\
$\mathrm{FV} \mathrm{dose}^{\mathrm{FV} \text { época }}$ & $0,247^{\mathrm{ns}}$ & & \\
$\mathrm{FV}$ dose x época & $2,190^{\mathrm{ns}}$ & & \\
$\mathrm{CV}(\%)$ & $1,052^{\mathrm{ns}}$ & & \\
\hline
\end{tabular}

\footnotetext{
${ }^{1}$ Médias seguidas de mesma letra maiúscula na coluna e minúscula na linha não diferem entre si para o teste Tukey a 0,05 de significância.

ns= Não significativo **significativo ao nível de $5 \%$ de probabilidade pelo teste $\mathrm{F}$.
} 
A altura média da inserção da espiga ficou entre 1,49 a 1,60 m. O suprimento inadequado de nitrogênio é considerado um fator limitante ao rendimento de grãos do milho, sendo que o $\mathrm{N}$ exerce uma importante função em processos bioquímicos da planta e determina o desenvolvimento das plantas de milho com aumento significativo na área foliar e na produção de massa de matéria seca (TAIZ ; ZEIGER, 2009; OLIVEIRA et al., 2009).

Neste sentido, grande parte dos trabalhos científicos relacionados na literatura avaliam o milho para a produção de grãos e em menor proporção com a finalidade da produção de massa para ensilagem. Meira et al. (2009) não verificaram diferenças significativas em relação à altura média da inserção da espiga em avaliação de diferentes fontes de $\mathrm{N}$ com diferentes tipos de parcelamento. Cardoso et al. (2011) também não observaram diferenças para a altura média de inserção de espigas em função de diferentes fontes de fertilizantes utilizados, incluindo a uréia.

Em relação à altura das plantas (TABELA 3) foram verificadas diferenças significativas para a fonte de variação (FV) época. As diferentes dosagens aplicadas não evidenciaram diferenças significativas, bem como a interação entre dose e época. As doses aplicadas de 100, 150 e $250 \mathrm{~kg} \mathrm{ha}^{-1}$ não apresentaram diferenças significativas em relação a época de aplicação, entretanto a aplicação de dosagem de $200 \mathrm{~kg} \mathrm{ha}^{-1}$ apresentou diferença em relação a altura média das plantas de milho. A aplicação que foi realizada aos 28 dias após o plantio apresentou o melhor desenvolvimento em relação à aplicada aos 35 dias após o plantio. As plantas que receberam a adubação aos 28 dias apresentaram um incremento médio de $19 \mathrm{~cm}$ em relação àquelas que receberam a aplicação aos 35 dias. A aplicação realizada aos 21 dias após o plantio não diferiu das demais épocas de aplicação.

Tabela 3 - Altura das plantas em função das diferentes doses de $\mathrm{N}$ e diferentes épocas de aplicação pósplantio.

\begin{tabular}{|c|c|c|c|}
\hline \multirow[t]{3}{*}{ Dose de $N$} & \multicolumn{3}{|c|}{ Altura Média das plantas (m) } \\
\hline & $\mathrm{Dl}$ & LANTIO & \\
\hline & 21 & 28 & 35 \\
\hline $100 \mathrm{~kg} \mathrm{ha}^{-1}$ & $2,88 \mathrm{Aa}$ & $2,96 \mathrm{Aa}$ & $2,86 \mathrm{Aa}$ \\
\hline $150 \mathrm{~kg} \mathrm{ha}^{-1}$ & $2,92 \mathrm{Aa}$ & $2,90 \mathrm{Aa}$ & $2,80 \mathrm{Aa}$ \\
\hline $200 \mathrm{~kg} \mathrm{ha}^{-1}$ & $2,85 \mathrm{Aab}$ & $2,97 \mathrm{Aa}$ & $2,78 \mathrm{Ab}$ \\
\hline $250 \mathrm{~kg} \mathrm{ha}^{-1}$ & $2,84 \mathrm{Aa}$ & $2,80 \mathrm{Aa}$ & 2,71Aa \\
\hline FV dose & $2,653^{\mathrm{ns}}$ & & \\
\hline FV época & $5,398 * *$ & & \\
\hline FV dose $x$ época & $0,636^{\mathrm{ns}}$ & & \\
\hline $\mathrm{CV}(\%)$ & 3,68 & & \\
\hline
\end{tabular}

${ }^{1}$ Médias seguidas de mesma letra maiúscula não diferem entre si na coluna e minúsculas não diferem em relação aos dias após o plantio dentro de cada dose de $\mathrm{N}$ na linha ao nível de $(\mathrm{p} \leq 0,05)$ para o teste Tukey. ns= Não significativo $* *$ significativo ao nível de $5 \%$ de probabilidade pelo teste $\mathrm{F}$.

Cardoso et al. (2011) avaliando diferentes fontes nitrogenadas não verificaram diferenças significativas para a altura do híbrido testado. Os resultados verificados neste trabalho são corroborados por Soratto et al. (2011) ao avaliar a utilização da uréia e duas fontes alternativas de $\mathrm{N}$ em cobertura, no milho sob plantio direto em solo arenoso. Entretanto, Cardoso et al. (2011) afirmaram que as alturas média da planta e da primeira espiga, sofreram influencia pela interação apresentada entre fonte e 
parcelamento, e que a aplicação parcelada da uréia, quando as plantas apresentavam 4 e 8 folhas expandidas, foi a que proporcionou plantas com a menor altura e segundo estes autores se deve a uma deficiência hídrica pós-aplicação que permitiu a volatilização de parte da uréia aplicada.

De acordo com Muzilli et al. (1989) entre 25 e 45 dias após a emergência, a planta de milho chega a acumular $43 \%$ do $\mathrm{N}$ que necessita e, entre as fases de desenvolvimento pleno, ainda absorve $31 \%$ das necessidades totais. Isso pode ser a causa de a aplicação realizada até os 35 dias após o plantio não apresentar diferenças significativas, uma vez que, o metabolismo da planta estaria no ponto máximo de absorção do nutriente.

Na Tabela 4 são apresentados os resultados referentes à produtividade média obtida em função dos diferentes tratamentos. Não foram verificadas diferenças significativas em relação à dose, época ou interação entre as duas variáveis.

Tabela 4 - Produtividade em função das diferentes doses de $\mathrm{N}$ e diferentes épocas de aplicação pósplantio.

\begin{tabular}{lccc}
\hline Dose de N & $\mathbf{3}$ & DIAS APÓS O PLANTIO \\
& \multicolumn{3}{c}{$\begin{array}{c}\text { Produtividade média } \\
\left(\mathrm{t} \mathrm{ha}^{-1}\right)\end{array}$} \\
\cline { 2 - 4 } & 21 & $48,67 \mathrm{Aa}$ & 35 \\
\hline $100 \mathrm{~kg} \mathrm{ha}^{-1}$ & $45,80 \mathrm{Aa}$ & $47,89 \mathrm{Aa}$ & $50,12 \mathrm{Aa}$ \\
$150 \mathrm{~kg} \mathrm{ha}^{-1}$ & $49,85 \mathrm{Aa}$ & $50,96 \mathrm{Aa}$ & $49,56 \mathrm{Aa}$ \\
$200 \mathrm{~kg} \mathrm{ha}^{-1}$ & $46,89 \mathrm{Aa}$ & $47,74 \mathrm{Aa}$ & $46,98 \mathrm{Aa}$ \\
$250 \mathrm{~kg} \mathrm{ha}^{-1}$ & $48,79 \mathrm{Aa}$ & $47,36 \mathrm{Aa}$ \\
$\mathrm{FV} \mathrm{dose}^{\mathrm{ns}}$ & & \\
FV época & $0,309^{\mathrm{ns}}$ & \\
$\mathrm{FV}$ dose x época & $0,422^{\mathrm{ns}}$ & \\
$\mathrm{CV}(\%)$ & $1,528^{\mathrm{ns}}$ & \\
\hline
\end{tabular}

${ }^{1}$ Médias seguidas de mesma letra maiúscula não diferem entre si na coluna e minúsculas não diferem em relação aos dias após o plantio dentro de cada dose de $\mathrm{N}$ na linha ao nível de $(\mathrm{p} \leq 0,05)$ para o teste Tukey. ns= Não significativo $* *$ significativo ao nível de $5 \%$ de probabilidade pelo teste $\mathrm{F}$.

Os resultados verificados para o híbrido avaliado permitem inferir que este híbrido apresenta adaptabilidade edafoclimática e características genéticas muito favoráveis para a região com produtividade média de massa para ensilagem acima de 46 toneladas por hectare.

Segundo, CSFEMG (1999), para uma produção entre 40 a $50 \mathrm{t} \mathrm{ha}^{-1}$ seriam necessários a aplicação em um solo de boa fertilidade níveis de 10 a $20 \mathrm{~kg}$ de N, $50 \mathrm{~kg}$ de $\mathrm{P}_{2} \mathrm{O}_{5}$ e $80 \mathrm{~kg} \mathrm{ha}^{-1}$ de $\mathrm{K}_{2} \mathrm{O}$ no plantio e uma cobertura com $130 \mathrm{~kg} \mathrm{ha}^{-1}$ de $\mathrm{N}$.

Estas recomendações são corroboradas nos resultados verificados no presente trabalho, sendo possível observar que para a condição de fertilidade do local do experimento o híbrido não apresentou respostas em doses superiores àquelas recomendadas, com a obtenção de produtividade estimada no planejamento de adubação para a cultura. As observações de Meira et al. (2009) também reiteram que a cultivar de milho apresenta interferência direta nestas correlações de fontes e épocas de aplicação, uma vez que existem híbridos responsivos e eficientes comparados a outros que não são responsivos ou apresentam menor eficácia em conversão do $\mathrm{N}$ aplicado. 


\section{CONCLUSÃO}

O híbrido AG 1051 apresentou produtividade estimada equivalente à dose recomendada pela literatura para o estado de Minas Gerais.

A melhor época de aplicação verificada foi aos 28 dias, correspondendo à dose de $200 \mathrm{~kg} \mathrm{ha}^{-1}$ de N.

A adubação de cobertura para o milho AG 1051 para a produção de silagem pode ser efetuada entre 21 a 35 dias após o plantio, em condições similares a do presente estudo.

\section{REFERÊNCIAS}

ALVES, V.M.C.et al. Milho. In: COMISSÃO DE FERTILIDADE DO SOLO DO ESTADO DE MINAS GERAIS (CFSEMG). Recomendação para o uso de corretivos e fertilizantes em Minas Gerais: $\mathbf{5}^{\circ}$ Aproximação. Viçosa, MG, 1999. 359 p.

CONAB - Companhia Brasileira de Abastecimento. Disponível em www.conab.gov.br ; acesso em: 30 set. 2010 ás $16 \mathrm{~h}: 45 \mathrm{~m}$.

FANCELLI, A.L.; LIMA, U.A. Milho: produção, pré-processamento e transformação agroindustrial. São Paulo: SICCI; PROMOCET; FEALQ, 1989. 112p. (Série Extensão Agroindustrial, 5)

FANCELLI, A. L.; DOURADO NETTO, D. Produção de milho. Guaíba: Agropecuária, 2000.h c6af..pdf

FERREIRA, D.F. SISVAR 5.0. Sistema de Análises Estatísticas. Lavras: UFLA, 2007.

MEIRA, F.A.et al. Fontes e épocas de aplicação do nitrogênio na cultura do milho irrigado. Ciências Agrárias, Londrina, v. 30, n. 2, p. 275-284, abr./jun. 2009.

MUZILli, O.; OLIVEIRA, E.L.; CALEGARI, A. Adubação do milho. Campinas: Cargill, v.4, 1989. $28 \mathrm{p}$.

OLIVEIRA, F.A.et al. Crescimento do milho adubado com nitrogênio e fósforo em um Latossolo Amarelo. Revista Brasileira de Ciências Agrárias, v.4, n.3, p.238-244, 2009.

POTAFOS. Brasil: consumo aparente de fertilizantes. Disponível em: <www.potafos.org>. Acesso em: 30 set. 2010 às $17 \mathrm{~h}: 30 \mathrm{~m}$.

SORATTO, R.P.et al. Doses e fontes alternativas de nitrogênio no milho sob plantio direto em solo arenoso. Ciência e Agrotecnologia, Lavras, v. 35, n. 1, p. 62-70, jan./fev. 2011.

TAIZ, L.; ZEIGER, E. Fisiologia vegetal. 3.ed. Porto Alegre: Artmed, 2009. 719p.

TOSELLO, G.A. Milhos especiais e seu valor nutritivo,In: VIEGAS, G. P.; PATERNIANE, E. (Ed.).Melhoramento e produção do milho. 2. ed.1987

YAMADA, T. Adubação nitrogenada do milho. Quanto, como e quando aplicar? Informações Agronômicas, Piracicaba: Potafos, n.74, p.1-5, 1996. 